\title{
THE PERRON INTEGRAL AND EXISTENCE AND UNIQUENESS THEOREMS FOR A FIRST ORDER NONLINEAR DIFFERENTIAL EQUATION
}

\author{
MANOUG N. MANOUGIAN ${ }^{1}$
}

\begin{abstract}
The Perron integral is used to establish an existence and uniqueness theorem concerning the initial value problem $y^{\prime}(t)=f(t, y(t))$, and $y\left(t_{0}\right)=\alpha$, for $t$ on the interval $I=\{t \mid 0 \leqq t \leqq 1\}$. The existence and uniqueness of the solution is obtained by use of a generalized Lipschitz condition, and a Picard sequence which is equiabsolutely continuous on $I$. Also, we prove a theorem on the uniqueness of solution by a generalization of Gronwall's inequality.
\end{abstract}

1. Introduction. This paper deals with existence and uniqueness theorems concerning the i.v.p. (initial value problem)

$$
y^{\prime}(t)=f(t, y(t)), \quad y\left(t_{0}\right)=\alpha,
$$

where $f(t, y(t))$, for any continuous $y$ on $I=\{t \mid 0 \leqq t \leqq 1\}$, is defined a.e. (almost everywhere) on $I$. There is an extensive body of literature dealing with conditions under which solutions for (1) exist. In most discussions $f$ is taken to be integrable in the Lebesgue sense. Here we use the Perron integral. It was shown by Bauer in [1] (see also Kamke [5], McShane [8], Saks [10]) that the Perron definition of the integral leads to a generalization of the Lebesgue integral. Northcutt [9] used the Perron integral, and obtained solutions for (1). The author [6] has also considered the Perron integral, and established an existence and uniqueness theorem for a second order nonlinear partial differential equation.

2. Preliminary theorems. Integration throughout this paper is in the Perron sense and the following theorems will be used.

Theorem 2.1 (Kamke [5, p. 210]). If $f \subset P$ (Perron integrable) on I and $f(t) \geqq 0$ a.e. on $I$, then, $f \subset \mathfrak{L}$ (Lebesgue integrable) on I, and for $t$ on $I, \int_{0}^{t} f=(\mathfrak{L}) \int_{0}^{t} f$.

Corollary 2.1.1. If $f$ and $g \subset P$ on $I$ and $f(t) \geqq g(t)$ a.e. on $I$, then, for $0 \leqq t_{0}<t \leqq 1, \int_{t_{0}}^{t} f \geqq \int_{t_{0}}^{t} g$.

Received by the editors June 27, 1969.

A MS Subject Classifications. Primary 3404, 3490; Secondary 2645.

Key Words and Phrases. Initial value problem, Lebesgue integral, Perron integral, bounded variation, Picard sequence, locally absolutely continuous, equicontinuous, equiabsolutely continuous, Cauchy-Euler methcd, Gronwall inequality.

1 The author wishes to express his thanks to Professor H. J. Ettlinger for his valuable contributions which led to this research. 
Corollary 2.1.2. If $f \subset P$ and $g \subset \mathfrak{L}$ on $I$ and $f(t) \geqq g(t)$ a.e. on $I$, then $f \subset \mathfrak{L}$ on $I$.

Theorem 2.2. If $f \subset P$ on $I$ and $g \subset \mathrm{B} . \mathrm{V}$. (bounded variation) on $I$ then $f \cdot g \subset P$ on $I$ and $\int_{0}^{t} f \cdot g=F(t) g(t)-\int_{0}^{t} F d g(s)$ where $F(t)=\int_{0}^{t} f$.

See Gordon and Lasher [3] for an elementary proof.

3. Existence theorems. We prove the following

THEOREM 3.1. If

H1. $f(t, y)$ is continuous in $y$ for $t$ a.e. on $I$.

H2. $f(t, y(t)) \subset P$ on I for $y$ continuous on $I$.

H3. $f(t, y(t)) \geqq g(t)$ a.e. on I where $g \subset P$ on $I$.

H4. $\left|f(t, y(t))-f\left(t, y^{*}(t)\right)\right| \leqq v(t)\left|y(t)-y^{*}(t)\right|$ a.e. on I where $v \subset P$ (and hence in $\mathcal{L}$ ) on $I$. Then there exists for the i.v.p. (1), a Picard sequence, which yields a solution, $\psi(t)$, which is continuous and locally absolutely continuous (LAC) on I, only if the sequence $\left\{\int_{0}^{t}\left(f_{n}-g\right)\right\}$ is EAC (equiabsolutely continuous) on $I$.

LEMMA 3.1. If

H1. $f_{n} \subset P$ on I for each counting number $n$.

H2. $\lim _{n} f_{n}(t)=f(t)$ a.e. on $I$.

H3. $f_{n}(t) \geqq g(t)$ a.e. on I for each $n$ where $g \subset P$ on $I$. Then, $f \subset P$ on $I$, and $\lim _{n} \int_{0}^{t} f_{n}=\int_{0}^{t} f$ only if the sequence $\left\{\int_{0}^{t}\left[f_{n}-g\right]\right\}$ is EAC on $I$.

The proof of this lemma (see [7]) is based on a corresponding theorem by Vitali [11] for functions integrable in the Lebesgue sense. Briefly, by Theorem 2.2, $\left(f_{n}-g\right) \subset \mathfrak{L}$ on $I$ and from [11]

$$
\lim _{n}(\mathfrak{L}) \int_{0}^{t}\left[f_{n}-g\right]=(\mathscr{L}) \int_{0}^{t}[f-g] .
$$

Consequently, $(f-g) \subset P$, and $\lim _{n} \int_{0}^{t}\left[f_{n}-g\right]=\int_{0}^{t}[f-g]$ and since $g \subset P$ on $I$, then $f \subset P$ on $I$ and $\lim _{n} \int_{0}^{t} f_{n}=\int_{0}^{t} f$.

Proof of Theorem 3.1. Let $y_{0}(t)=\alpha$;

$$
y_{n+1}(t)=\int_{t_{0}}^{t} f\left(s, y_{n}(s)\right) d s+\alpha \quad(n=0,1,2, \cdots) .
$$

We note that $y_{1}(t)$ is continuous and LAC (see Saks [10, p. 251]) on $I$. By induction, we have that $y_{n}(t)$ is continuous and LAC on $I$ for each counting number $n$. Define

$$
u_{n}(t)=y_{n+1}(t)-y_{n}(t) \quad(n=0,1,2, \cdots) .
$$

Then, $u_{0}(t)=\int_{t_{0}}^{t} f(s, \alpha) d s$ and 


$$
u_{n}(t)=\int_{t_{0}}^{t}\left[f\left(s, y_{n}(s)\right)-f\left(s, y_{n-1}(s)\right)\right] d s .
$$

Under $\mathrm{H} 4$, the integral in (4) may be taken in the Lebesgue sense. Now, since $u_{0}(t)$ is continuous on $I$, then there exists a number $k$ such that for $t$ on $I .\left|u_{0}(t)\right|<k$ and $\left|u_{1}(t)\right|<k \int_{0}^{t} v$ and in general, since $v(t) \geqq 0$ a.e. on $I$

$$
\left|u_{n}(t)\right|<k\left[\int_{0}^{t} v\right]^{n} / n ! \leqq k\left[\int_{0}^{1} v\right]^{n} / n ! .
$$

Consequently, $\quad \sum_{i=0}\left|u_{i}(t)\right|$ converges uniformly on $I$. But, $\sum_{i=0}^{n} u_{i}(t)=y_{n+1}(t)-y_{0}(t)$. Hence, there exists a function $\psi(t)$ such that $\lim _{n} y_{n}(t)=\psi(t)$ uniformly on $I$ where $\psi(t)$ is continuous and LAC on $I$. From $\mathrm{H} 1$ we have $\lim _{n} f\left(t, y_{n}(t)\right)=f(t, \psi(t))$ a.e. on $I$.

Then, H3, and Lemma 3.1, yield

$$
\psi(t)=\int_{t_{0}}^{t} f(s, \psi(s)) d s+\alpha
$$

where $\psi\left(t_{0}\right)=\alpha$ and $\psi^{\prime}(t)=f(t, \psi(t))$ a.e. on $I$. Q.E.D.

Under the hypotheses $\mathrm{H} 1-\mathrm{H} 3$ of Theorem 3.1, and by use of the Cauchy-Euler method, Northcutt [9] showed that there exists a function $\psi(t)$, continuous and LAC, which is a solution of the i.v.p. (1) only if the sequence $\left\{\int_{0}^{t}\left[f_{n}-g\right]\right\}$ is EAC on $I$. His method of proof is based on Ascoli's theorem on a uniformly bounded set of equicontinuous functions on $I$, and on Lemma 3.1. Uniqueness is not to be expected in this case.

\section{Uniqueness theorems.}

Theorem 4.1. The solution $\psi(t)$ in Theorem 3.1 is unique.

Proof. Assume that there exists for the i.v.p. (1) another solution $\psi^{*}(t)$. Let $Y(t)=\psi(t)-\psi^{*}(t)$ for $t$ on $I$. Then,

$$
Y(t)=\int_{t_{0}}^{t}\left[f(s, \psi(s))-f\left(s, \psi^{*}(s)\right)\right] d s
$$

and

$$
0 \leqq|Y(t)| \leqq \lim _{n} k\left[(\mathscr{L}) \int_{0}^{1} v\right]^{n} / n !
$$

Hence $Y(t)=0$ for $t$ on $I$ and uniqueness of solution for (1) follows. The following involve generalizations of some of the results of Ettlinger [2]. 
THEOREM 4.2. If $\psi_{1}(t)$ and $\psi_{2}(t)$ are LAC on I and satisfy the i.v.p. (1) in a region $R=\{(t, y) \mid 0 \leqq t \leqq 1$, all $y\}$. If further, $\psi_{1}(t)$ and $\psi_{2}(t)$ satisfy $\mathrm{H} 2$ and $\mathrm{H} 4$ of Theorem 3.1 on I. Then, $\psi_{1}(t)=\psi_{2}(t)$ on $I$.

A proof of this theorem may be obtained by use of the following lemma, which is a generalization of Gronwall's lemma [4].

LemMa 4.2.1. If $h \subset \mathcal{L}$ and $g \subset P$ on $I, x$ is LAC on $I$, and satisfy the differential inequality

$$
x^{\prime}(t)+h(t) x(t) \leqq g(t) \text {, a.e. on } I .
$$

Then, for $0 \leqq t_{0}<t \leqq 1$,

$$
x(t) \leqq \exp \left(-\int_{t_{0}}^{t} h\right)\left[\int_{t_{0}}^{t} g \exp \left(\int_{t_{0}}^{s} h\right)+x\left(t_{0}\right)\right] .
$$

Proof. Since $\exp \left(\int_{t_{0}}^{t} h\right)>0$ we have

$$
x^{\prime}(t) \exp \left(\int_{t_{0}}^{t} h\right)+h(t) x(t) \exp \left(\int_{t_{0}}^{t} h\right) \leqq g(t) \exp \left(\int_{t_{0}}^{t} h\right) .
$$

Now, $g \subset P$ and $\exp \left(\int_{t_{0}}^{t} h\right)$ is absolutely continuous on $I$. Then, by Theorem 2.2, $g \cdot \exp \left(\int_{t_{0}}^{t} h\right) \subset P$ on $I$. Furthermore,

$$
\begin{aligned}
\frac{d}{d l}\left[x(t) \exp \left(\int_{t_{0}}^{t} h\right)\right]=x^{\prime}(t) & \exp \left(\int_{t_{0}}^{t} h\right) \\
& +h(t) x(t) \exp \left(\int_{t_{0}}^{t} h\right) \text { a.e. on } I .
\end{aligned}
$$

Hence, by Corollary 2.1.1, and (8), we have for $t \geqq t_{0}$

$$
\left[x(s) \exp \left(\int_{t_{0}}^{s} h\right)\right]_{t_{0}}^{t} \leqq \int_{t_{0}}^{t} g(s) \exp \left(\int_{t_{0}}^{s} h\right)
$$

and statement (7) is thus obtained. We note that the equality in (7), $\phi(t)=\exp \left(-\int_{t_{0}}^{t} h\right)\left[\int_{t_{0}}^{t} g \exp \left(\int_{t_{0}}^{s} h\right)+\phi\left(t_{0}\right)\right]$, represents a solution for the linear differential equation $x^{\prime}(t)+h(t) x(t)=g(t)$.

Corollary 4.2.1. If $x\left(t_{0}\right)=0$ and, a.e. on $I, g(t)=0$ and $x(t) \geqq 0$, then $x(t)=0$ on $\left[t_{0}, 1\right]$.

Proof of Theorem 4.2. Let $x(t)=\left|\psi_{1}(t)-\psi_{2}(t)\right|$ on $I$. Then, $x^{\prime}(t) \leqq v(t) x(t)$ a.e. on $I$. Since $x\left(t_{0}\right)=0$, then for $t \leqq t_{0}$ and by Corollary 2.1.1

$$
x(t) \geqq \int_{t_{0}}^{t} v x
$$


Now, from $H 4$ and by Corollary 2.1.2, $\left|f\left(t, \psi_{1}(t)\right)-f\left(t, \psi_{2}(t)\right)\right| \subset P$ on $I$, and is also integrable in the Lebesgue sense. Hence, for $t$ on $I$, $\mathrm{H} 4$ yields

$$
x(t) \leqq \int_{t_{0}}^{t} v x
$$

From (10) and (11) we have on $\left[0, t_{0}\right]$

$$
x(t)=\int_{t_{0}}^{t} v x
$$

and $x^{\prime}(t)=v(t) x(t)$ a.e. on $\left[0, t_{0}\right]$. Corollary 4.2.1 then yields

$$
x(t)=0 \text { on } I \text {. }
$$

Hence, $\psi_{1}(t)=\psi_{2}(t)$ on $I$.

5. Remark. In equation (1), $y(t)$ may be considered as a real valued vector function defined in a Euclidean space of $n$ dimensions. Conclusions follow as in the scalar case.

\section{REFERENCES}

1. H. Bauer, Der Perronsche Integralhegriff und seine Beziehung zum Lebesgueshen, Monatsh. Math. und Physik 26 (1915), 153-198.

2. H. J. Ettlinger, Linear derivative inequalities and differential equations, National Math. Mag. 11 (1936), 1-5.

3. L. Gordon and S. Lasher, An elementary proof of integration by parts for the Perron integral, Proc. Amer. Math. Soc. 18 (1967), 394-398. MR 35 \#1726.

4. T. H. Gronwall, Note on the derivatives with respect to a parameter of the solutions of a system of differential equations, Ann. of Math. (2) 20 (1918/19), 292-296.

5. E. Kamke, Das Lebesgue-Stieltjes-Integral, Teubner Verlagsgesellschaft, Leipzig, 1956. MR 18, 384.

6. M. N. Manougian, An existence and uniqueness theorem for a nonlinear differential equation, J. Differential Equations (to appear).

7. - On the convergence of a sequence of Perron integrals, Proc. Amer. Math. Soc. 23 (1969), 320-332.

8. E. J. McShane, Integration, Princeton Univ. Press, Princeton, N. J., 1944. MR 6, 43.

9. R. A. Northcutt, Perron type differential and integral inequalities, Dissertation, The University of Texas, Austin, Texas, 1968.

10. S. Saks, Theory of the integral, 2nd rev. ed., Dover, New York, 1964. MR 29 \#4850.

11. G. Vitali, Sul integrazione per serie, Rend. Circ. Mat. Palermo 23 (1907), $137-155$.

University of South Florida, Tampa, Florida 33620 